\title{
Bio-Inspired Sensor Design with an Array of Coupled Lasers
}

\author{
Wenxue Wang and Bijoy K. Ghosh
}

\begin{abstract}
The basic idea of the paper is to use neurocomputational features of weakly connected neural networks of oscillators in pattern recognition. Stable synchronized states of the neural networks where artificial neurons are lasers with equal natural frequencies are prespecified with connection matrices. A set of memorized patterns are associated with the prescribed equilibria in phase relations with which neurons oscillate. Any pattern to be recognized in the neighborhood of the memorized patterns will reach the corresponding stable synchronized state. Phase locking takes part in the recognition mechanism. Kuramoto's model is used in designing equilibria of laser networks.
\end{abstract}

Index Terms-Neural Network, Oscillator, Laser, Pattern Recognition.

\section{INTRODUCTION}

A novel computing paradigm, emerging from a network of artificial neural oscillators, has been studied [1] [2] [8]. Typically, simple analog units, artificial neurons, in the network process information in parallel. Such neural networks perform pattern recognition and associative recall via selforganization of neurons.

One candidate network is the weakly connected neural networks where artificial neurons are identical coupled lasers. With such a network, the possibility that the laser oscillators interact via phases, which corresponds to the phase modulation (PM) encoding, was proposed [9]. The approach is in the spirit of FM interaction theory.

In this paper, we propose a different phase modulation encoding mechanism with such neural network for the purpose of decoding from a visual scene. Elements of the oscillator network interact with each other via phases, rather than amplitudes and memorized patterns correspond to synchronized states where each unit of the oscillator network oscillate with equal frequencies and with prescribed phase relations. These equilibria in phase relations are prespecified with connection coefficients among oscillatory units. The mechanism of recognition is related to phase locking.

One of the applications of the mechanism of recognition with the coupled laser networks is to decode or detect visual inputs from cortical waves in visual cortices of freshwater turtles. A large-scale model of turtle visual cortex was used to study the dynamics of propagating waves in the cortex, and detection and estimation of visual inputs [11] [12] [4] [14] [15]. Oscillatory neural networks of coupled lasers could provide a new technique.

W. Wang and B. K. Ghosh are with the Department of Electrical and Systems Engineering, Washington University in Saint Louis, Saint Louis, MO 63130, USA $\{w w 1$, ghosh\}@netra.wust1.edu

\section{THE MOdel OF A LASER NetWORK}

The network of coupled lasers has been described in detail [9] Below is the brief description. The lasers are written with the dimensionless rate equations as follows:

$$
\begin{gathered}
\dot{E}_{i}=(1+i \alpha) N_{i} E_{i}+i \omega E_{i}+\sum_{j=1}^{n} c_{i j} E_{j}, \\
\dot{N}_{i}=\mu\left[P-N_{i}-\left(1+2 N_{i}\right)\left|E_{i}\right|^{2}\right],
\end{gathered}
$$

where $E_{i}$ and $N_{i}$ are the complex electric field and the excess carrier number of the $i$ th laser, the derivatives ${ }^{\circ}=d / d s, s=$ $t \tau_{p}^{-1}$ is the time measured in units of the photon lifetime $\tau_{p}$, $\mu=\tau_{p} / \tau_{s}$ is the ratio of photon to carrier time scales, where $\tau_{s}$ is the carrier lifetime, $P$ is the pumping above threshold, $\alpha$ is the linewidth enhancement factor, $\omega$ is normalized optical frequency, and $c_{i j}$ are complex connection coefficients.

It is convenient to use polar coordinates $E_{i}=r_{i} e^{i \phi_{i}}$ and $c_{i j}=s_{i j} e^{i \psi_{i j}}$ to rewrite the model (1) and (2) in the form

$$
\begin{gathered}
\dot{\phi}_{i}=\alpha N_{i}+\omega+\sum_{j=1}^{n} s_{i j} \frac{r_{j}}{r_{i}} \sin \left(\phi_{j}+\psi_{i j}-\phi_{i}\right), \\
\dot{r}_{i}=N_{i} r_{i}+\sum_{j=1}^{n} s_{i j} r_{j} \cos \left(\phi_{j}+\psi_{i j}-\phi_{i}\right), \\
\dot{N}_{i}=\mu\left[P-N_{i}-\left(1+2 N_{i}\right)\left|r_{i}\right|^{2}\right] .
\end{gathered}
$$

In the case of weak connection, the dynamical analysis of the model (3),(4) and (5) shows that

$$
\left(r_{i}(t), N_{i}(t)\right) \longrightarrow(\sqrt{P}, 0),
$$

and the phase $\phi_{i}(t) \rightarrow \omega t+\phi_{i}^{0}$, where $\phi_{i}^{0}$ is determined by the initial conditions.

The phase differences, but not phases, play a key role in the neurocomputing mechanism proposed in this paper. To illustrate the main idea, we would like to review a model proposed by Kuramoto [10].

\section{Phase Locking With A Network OF Kuramoto MODELS}

If all $r_{i}(t) \rightarrow r_{0}$, then the phase model (3) for $\alpha=0$ has Kuramoto's form [10]

$$
\dot{\phi}_{i}=\omega+\sum_{j=1}^{n} s_{i j} \sin \left(\phi_{j}-\phi_{i}+\psi_{i j}\right)
$$

where $\phi_{i}, i=1, \cdots, n$ (assume $n=2$ for illustration), are phase variables taking values in the interval $[-\pi, \pi)$. The parameters $s_{i j}$ and $\psi_{i j}$ are assumed to satisfy $s_{12}=s_{21}$, $\psi_{12}=-\psi_{21}$. The index $i$, refers to the $i^{t h}$ unit and these 
units are coupled. In order to understand the dynamics of (6), we define a new variable $\phi=\phi_{1}-\phi_{2}$ and rewrite (6) as follows

$$
\dot{\phi}=-2 s_{12} \sin \left(\phi-\psi_{12}\right)
$$

The stationary points of (7) are given by $\phi-\psi_{12}=n \pi$, out of which the stable points are given precisely by

$$
\phi-\psi_{12}=2 n \pi, \quad n=0, \pm 1, \pm 2, \cdots
$$

For $\phi_{1}, \phi_{2}$ in the interval $[-\pi, \pi)$, we have $\phi=\psi_{12}$ and $\phi=\psi_{12}+2 \pi$ are the two stable points if $\psi_{12}<0$, and $\phi=\psi_{12}$ and $\phi=\psi_{12}-2 \pi$ are the two stable points if $\psi_{12}>0$. Up to $\bmod 2 \pi$, the two stable points of $\phi$ are actually the same indicating that (7) converges globally to an unique equilibrium point.

Memory with two elements: In order to use (7) for the purpose of memorizing an input pattern, we would require that it has multiple equilibria. This is achieved by rescaling the phase variables as follows:

$$
\bar{\phi}_{1}=\frac{1}{2} \phi_{1}, \quad \bar{\phi}_{2}=\frac{1}{2} \phi_{2}
$$

Rewriting (6) with respect to the new variables, we obtain

$$
\begin{aligned}
& \dot{\bar{\phi}}_{1}=\frac{1}{2} \omega+\frac{1}{2} s_{12} \sin \left(2 \bar{\phi}_{2}-2 \bar{\phi}_{1}+\psi_{12}\right) \\
& \dot{\bar{\phi}}_{2}=\frac{1}{2} \omega+\frac{1}{2} s_{21} \sin \left(2 \bar{\phi}_{1}-2 \bar{\phi}_{2}+\psi_{21}\right)
\end{aligned}
$$

By defining $\bar{\phi}=\bar{\phi}_{1}-\bar{\phi}_{2}$, analogously we obtain

$$
\dot{\bar{\phi}}=-s_{12} \sin \left(2 \bar{\phi}-\psi_{12}\right)
$$

If $\psi_{12}>0$, the two stable stationary points of (9) are given by $\frac{\psi_{12}}{2}$ and $\frac{\psi_{12}}{2}-\pi$ in the interval $[-\pi, \pi)$. The case, $\psi_{12}<0$, can be analogously described. Additionally it can be verified that if

$$
\frac{\psi_{12}}{2}-\frac{\pi}{2}<\bar{\phi}(0)<\frac{\psi_{12}}{2}+\frac{\pi}{2}
$$

then $\bar{\phi}(t)$ converges to the first stable point $\frac{\psi_{12}}{2}$. Otherwise it converges to $\frac{\psi_{12}}{2}-\pi$. In Fig. 1 we plot the phase variable $\bar{\phi}(t)$ as a unit complex number $e^{i \bar{\phi}(t)}$.

The main idea we would like to explore is to utilize the convergence properties of (9) to distinguish between a pair of complex patterns. Let us define the following two vectors in $\mathbb{C}^{2}$ as follows:

$$
p_{1}=\left(\begin{array}{c}
\pi_{1} \\
\pi_{2}
\end{array}\right), p_{2}=\left(\begin{array}{cc}
e^{-i \frac{\pi}{2}} & \pi_{1} \\
e^{+i \frac{\pi}{2}} & \pi_{2}
\end{array}\right)
$$

where $\pi_{1}$ and $\pi_{2}$ are any two complex numbers such that

$$
\left|\pi_{1}\right|=\left|\pi_{2}\right|=1
$$

and

$$
\arg \left(\pi_{1} \bar{\pi}_{2}\right)=\frac{\psi_{12}}{2}
$$

Define a mapping

$$
\xi: \mathbb{C}^{2} \longrightarrow \mathbb{R}
$$

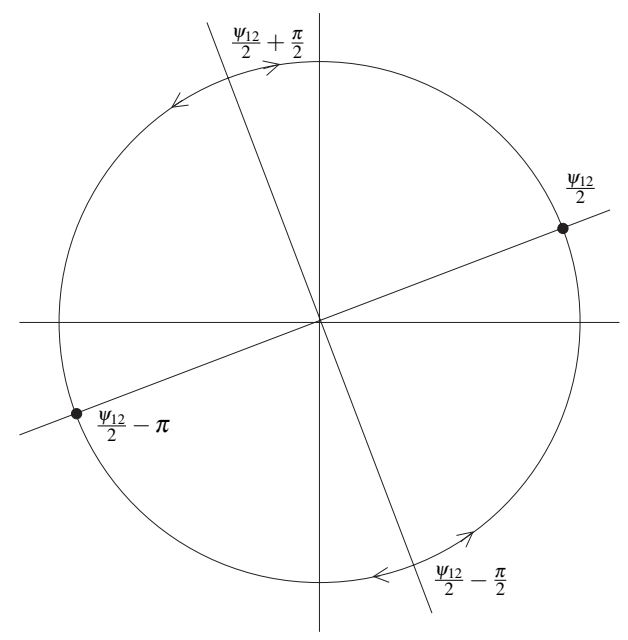

Fig. 1: Phase variable $\bar{\phi}(t)$ is plotted as an unit complex number $e^{i \bar{\phi}(t)}$ showing two stable equilibria.

as follows

$$
\left(\begin{array}{l}
w_{1} \\
w_{2}
\end{array}\right) \longmapsto \arg \left(w_{1} \bar{w}_{2}\right)
$$

It would follow that $\xi\left(p_{1}\right)=\frac{\psi_{12}}{2}$ and $\xi\left(p_{2}\right)=\frac{\psi_{12}}{2}-\pi$. Thus the two patterns $p_{1}$ and $p_{2}$ are mapped to the two stable equilibria of (9) under the map $\xi$. Patterns which are close to either $p_{1}$ or $p_{2}$ would be attracted towards the corresponding equilibrium. This principal can therefore be used as a memory.

Usually we are not interested in a set of complex patterns. Rather we would like to memorize a pattern of real vectors. Assume that we have pair of vectors in $\mathbb{R}^{2}$ which we would like to memorize. We consider a map

$$
\begin{aligned}
T & : \mathbb{R}^{2} \longrightarrow \mathbb{C}^{2} \\
& v_{1} \longmapsto p_{1} \\
& v_{2} \longmapsto p_{2}
\end{aligned}
$$

The dynamics of (9) can be used to memorize the two vectors $v_{1}$ and $v_{2}$. This point is illustrated by choosing $v_{1}=\left(\begin{array}{ll}1 & 0\end{array}\right)^{T}, v_{2}=\left(\begin{array}{ll}0 & 1\end{array}\right)^{T}$ and the transformation $T$ in (12) described as

$$
\begin{aligned}
& T: \mathbb{R}^{2} \longrightarrow \mathbb{C}^{2}
\end{aligned}
$$

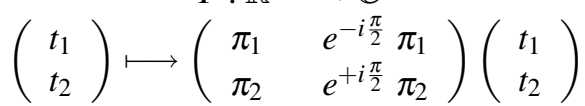

Combining the two maps (11) and (13) we obtain

$$
\xi T\left(\begin{array}{l}
t_{1} \\
t_{2}
\end{array}\right)=\arg \left(\left(t_{1}+t_{2} e^{-i \frac{\pi}{2}}\right)^{2} \pi_{1} \bar{\pi}_{2}\right)
$$

If we now assume that the vector $\left(\begin{array}{ll}t_{1} & t_{2}\end{array}\right)$ is chosen such that

$$
-\frac{\pi}{4}<\arg \left(t_{1}+t_{2} e^{-i \frac{\pi}{2}}\right)<\frac{\pi}{4}
$$

it would follow that

$$
\frac{\psi_{12}}{2}-\frac{\pi}{2}<\xi T\left(\begin{array}{c}
t_{1} \\
t_{2}
\end{array}\right)<\frac{\psi_{12}}{2}+\frac{\pi}{2}
$$


If $\bar{\phi}(t)$ is initialized at

$$
\bar{\phi}(0)=\xi T\left(\begin{array}{l}
t_{1} \\
t_{2}
\end{array}\right)
$$

we would conclude from (10) that $\bar{\phi}(t)$ converges to $\frac{\psi_{12}}{2}$. Thus all vector patterns that satisfy (14) would be categorized as pattern 1 . These are precisely given by

$$
\left|t_{1}\right|>\left|t_{2}\right| \text {. }
$$

Analogously all vectors that satisfy

$$
\left|t_{1}\right|<\left|t_{2}\right| \text {. }
$$

would be categorized as pattern 2 .

Memorizing more than two patterns: In order to memorize more than two patterns, for example 3 patterns, the phase variables can be rescaled as

$$
\bar{\phi}_{1}=\frac{1}{3} \phi_{1}, \quad \bar{\phi}_{2}=\frac{1}{3} \phi_{2}
$$

In this case we can rewrite (9) as

$$
\dot{\bar{\phi}}=-\frac{2}{3} s_{12} \sin \left(3 \bar{\phi}-\psi_{12}\right)
$$

and the stable stationary points are given by

$$
\frac{\psi_{12}}{3}, \frac{\psi_{12}}{3}+\frac{2 \pi}{3}, \frac{\psi_{12}}{3}+\frac{4 \pi}{3}
$$

assuming $\psi_{12}<0$. Analogous construction can be made with complex patterns in $\mathbb{C}^{2}$ given by

$$
p_{1}=\left(\begin{array}{c}
\pi_{1} \\
\pi_{2}
\end{array}\right), p_{2}=\left(\begin{array}{c}
e^{i \frac{\pi}{3}} \pi_{1} \\
e^{-i \frac{\pi}{3}} \pi_{2}
\end{array}\right), p_{3}=\left(\begin{array}{c}
e^{i \frac{2 \pi}{3}} \pi_{1} \\
e^{-i \frac{2 \pi}{3}} \pi_{2}
\end{array}\right)
$$

where

$$
\left|\pi_{1}\right|=\left|\pi_{2}\right|=\left|\pi_{3}\right|=1, \quad \arg \left(\pi_{1} \bar{\pi}_{2}\right)=\frac{\psi_{12}}{3} .
$$

The map $\xi$ can be chosen identical to (11) and the map $T$ is defined as

$$
\begin{gathered}
T: \mathbb{R}^{3} \longrightarrow \mathbb{C}^{2} \\
v_{i} \longmapsto p_{i}
\end{gathered}
$$

where $i=1,2,3$. In order to memorize vectors $\left(\begin{array}{lll}1 & 0 & 0\end{array}\right)^{T}$, $\left(\begin{array}{lll}0 & 1 & 0\end{array}\right)^{T}$, and $\left(\begin{array}{lll}0 & 0 & 1\end{array}\right)^{T}$, we define

$$
\left(\begin{array}{c}
t_{1} \\
t_{2} \\
t_{3}
\end{array}\right) \longmapsto\left(\begin{array}{ccc}
\pi_{1} & e^{i \frac{\pi}{3}} \pi_{1} & e^{i \frac{2 \pi}{3}} \pi_{1} \\
\pi_{2} & e^{-i \frac{\pi}{3}} \pi_{2} & e^{-i \frac{2 \pi}{3}} \pi_{2}
\end{array}\right)\left(\begin{array}{c}
t_{1} \\
t_{2} \\
t_{3}
\end{array}\right)
$$

The phase difference variable $\bar{\phi}$ is initialized at

$$
\bar{\phi}(0)=\xi T\left(\begin{array}{c}
t_{1} \\
t_{2} \\
t_{3}
\end{array}\right)
$$

If

$$
-\frac{\pi}{6}<\arg \left(t_{1}+t_{2} e^{i \frac{\pi}{3}}+t_{3} e^{i \frac{2 \pi}{3}}\right)<\frac{\pi}{6}
$$

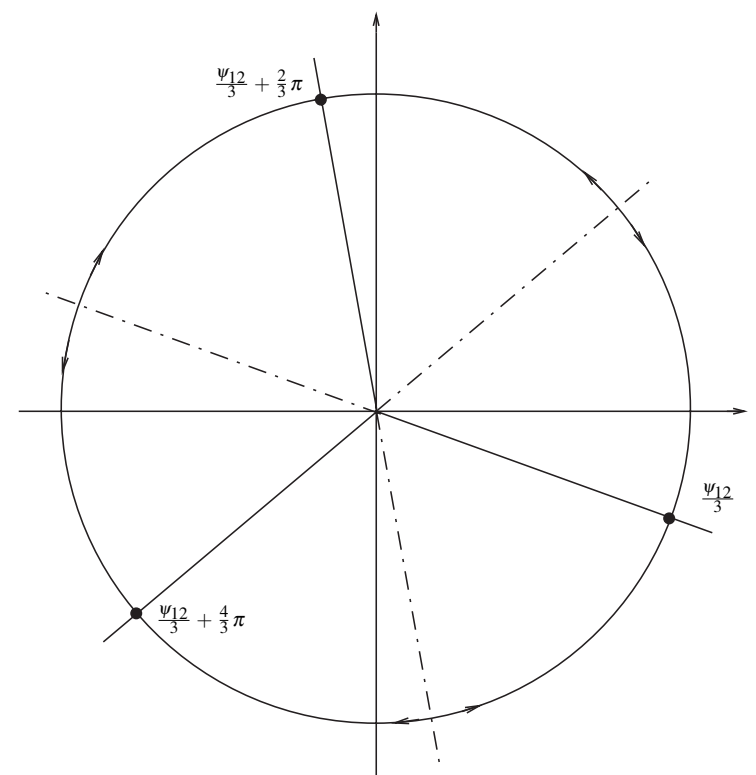

Fig. 2: In this figure we show the three regions of convergences for the dynamical system (15) under initial condition (16). Each region has been indicated by the argument of $t_{1}+t_{2} e^{i \pi / 3}+t_{3} e^{2 i \pi / 3}$.

then $\bar{\phi}(t)$ converges to $\frac{\psi_{12}}{3}$. All the other regions of convergences have been sketched in Fig. 2.

Memorizing patterns with third order dynamics: Another way to memorize more than two patterns is to use a higher order Kuramoto model that introduces multiple equilibria. One way to achieve this is to consider the following third order dynamics:

$$
\begin{aligned}
& \dot{\phi}_{1}=\omega+s_{12} \sin \left(\phi_{2}-\phi_{1}+\psi_{12}\right)+s_{13} \sin \left(\phi_{3}-\phi_{1}+\psi_{13}\right) \\
& \dot{\phi}_{2}=\omega+s_{21} \sin \left(\phi_{1}-\phi_{2}+\psi_{21}\right)+s_{23} \sin \left(\phi_{3}-\phi_{2}+\psi_{23}\right) \\
& \dot{\phi}_{3}=\omega+s_{31} \sin \left(\phi_{1}-\phi_{3}+\psi_{31}\right)+s_{32} \sin \left(\phi_{2}-\phi_{3}+\psi_{32}\right)
\end{aligned}
$$

where as before we assume $s_{i j}=s_{j i}$ and $\psi_{i j}=-\psi_{j i}$. Let us now define the phase difference variables as follows:

$$
\phi_{i j}=\phi_{i}-\phi_{j}
$$

and obtain $\left[\dot{\phi}_{12}, \dot{\phi}_{23}, \dot{\phi}_{31}\right]^{T}$ as

$$
\left[\begin{array}{ccc}
-2 s_{12} & s_{23} & s_{31} \\
s_{12} & -2 s_{23} & s_{31} \\
s_{12} & s_{23} & -2 s_{31}
\end{array}\right]\left[\begin{array}{c}
\sin \left(\phi_{12}-\psi_{12}\right) \\
\sin \left(\phi_{23}-\psi_{23}\right) \\
\sin \left(\phi_{31}-\psi_{31}\right)
\end{array}\right] .
$$

Up to $\bmod 2 \pi$, the above equation has a unique stable stationary point at $\psi_{12}, \psi_{23}, \psi_{31}$. Scaling the phase variables as

$$
\bar{\phi}_{1}=\frac{1}{2} \phi_{1}, \bar{\phi}_{2}=\frac{1}{2} \phi_{2}, \bar{\phi}_{3}=\frac{1}{2} \phi_{3},
$$

we can write down the dynamics of $\bar{\phi}_{12}, \bar{\phi}_{23}, \bar{\phi}_{31}$ where $\bar{\phi}_{i j}=\bar{\phi}_{i}-\bar{\phi}_{j}$. The four stable stationary points are given by

$$
\left(\begin{array}{c}
\frac{\psi_{12}}{2} \\
\frac{\psi_{23}}{2} \\
\frac{\psi_{12}+\psi_{23}}{2}
\end{array}\right),\left(\begin{array}{c}
\frac{\psi_{12}}{2}+\pi \\
\frac{\psi_{23}}{2} \\
\frac{\psi_{12}+\psi_{23}}{2}+\pi
\end{array}\right)
$$




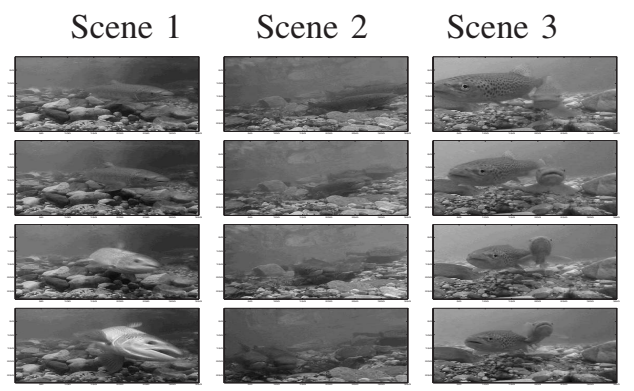

Fig. 3: Each column represents 4 sequential images of a scene. Three different scenes are shown here.

$$
\left(\begin{array}{c}
\frac{\psi_{12}}{2} \\
\frac{\psi_{23}}{2}+\pi \\
\frac{\psi_{12}+\psi_{23}}{2}+\pi
\end{array}\right),\left(\begin{array}{c}
\frac{\psi_{12}}{2}+\pi \\
\frac{\psi_{23}}{2}+\pi \\
\frac{\psi_{12}+\psi_{23}}{2}
\end{array}\right) .
$$

Four patterns of vectors in $\mathbb{C}^{3}$ are defined as follows:

$$
\begin{gathered}
P_{1}=\left(\begin{array}{c}
\pi_{1} \\
\pi_{2} \\
\pi_{3}
\end{array}\right), P_{2}=\left(\begin{array}{c}
e^{\frac{i \pi}{2}} \pi_{1} \\
e^{\frac{-i \pi}{2}} \pi_{2} \\
e^{\frac{-i \pi}{2}} \pi_{3}
\end{array}\right) \\
P_{3}=\left(\begin{array}{c}
e^{\frac{i \pi}{2}} \pi_{1} \\
e^{\frac{i \pi}{2}} \pi_{2} \\
e^{\frac{-i \pi}{2}} \pi_{3}
\end{array}\right), P_{4}=\left(\begin{array}{c}
e^{\frac{i \pi}{2}} \pi_{1} \\
e^{\frac{-i \pi}{2}} \pi_{2} \\
e^{\frac{i \pi}{2}} \pi_{3}
\end{array}\right)
\end{gathered}
$$

where we assume that

$$
\psi_{12}=2\left(\arg \pi_{1} \bar{\pi}_{2}\right), \psi_{23}=2\left(\arg \pi_{2} \bar{\pi}_{3}\right)
$$

For here onwards, it is quite easy to define a transformation that would map $\mathbb{R}^{4}$ to $\mathbb{C}^{3}$ and would be defined in such a way that $u_{i}$ is mapped to $P_{i}$, where $u_{i}$ is the $i^{t h}$ standard unit vector.

\section{Two Illustrative Examples:}

In this section we use two illustrative examples to show how Kuramoto model can be used to memorize three patterns.

\section{A. Decoding from a sequence of visual images}

The convergence properties of the Kuramoto model can be easily linked with the task of recognizing scenes such as the one in Fig. 3 used as visual input to the cortex model [15]. Each scene has already been encoded as a vector in $\mathbb{R}^{60}$ that was used as a cortical input. An initial estimate of the cortical input is provided by the ARMA models [5]. In this section we propose to obtain a refined estimate using the Kuramoto model. Our task is to distinguish between 3 patterns along any horizontal line in Fig. 3. This would therefore be an alternative to the one described so far utilizing the ARMA models This is achieved by defining a map

$$
T: \mathbb{R}^{60} \longrightarrow \mathbb{C}^{M}
$$

which maps

$$
v_{i} \longmapsto p_{i}
$$

where $M=2$ or 3 (in case of the networks of 2 or 3 oscillatory units), $v_{1}, v_{2}$, and $v_{3}$ are the three associated

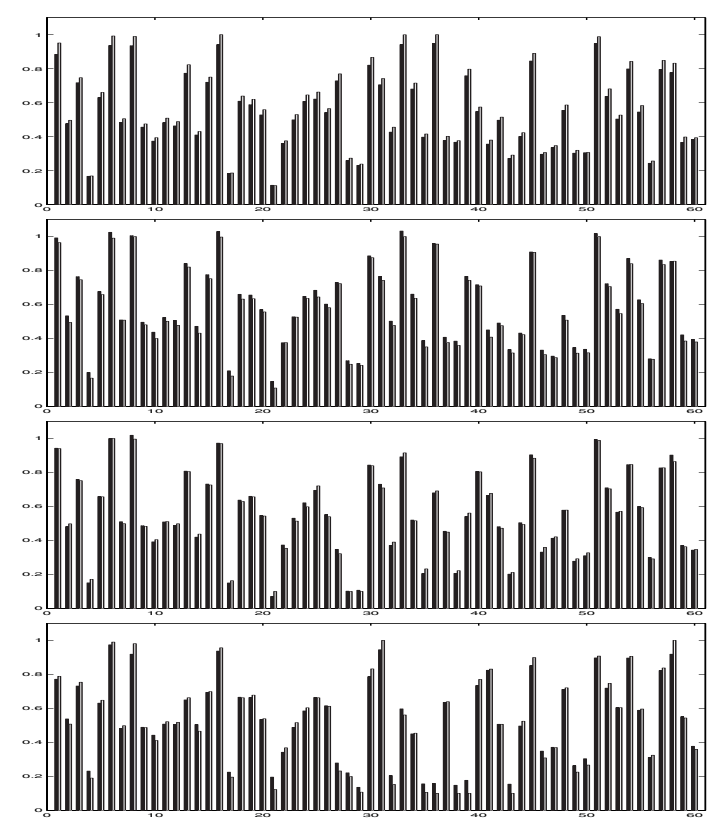

Fig. 4: Scene 1 in the above Fig. 3 is sparsely coded by $60-$ dimension vectors. The vectors are shown here for each of the images in scene 1. The blank bars are the actual vectors and the black bars are the estimated vectors

encoding of the vectors in $\mathbb{R}^{60}$ for the top three images in Fig. 3. For each of the other 3 horizontal axis of Fig. 3, the corresponding triplet of estimated vectors are mapped as an initial condition of $\bar{\phi}(t)$ and the recognition results with third order Kuramoto model are plotted in Fig. 5. The point of this illustration is that the Kuramoto model can be used to track a moving scene. Every image of the scene has been correctly classified and they all fall within the region of attraction.

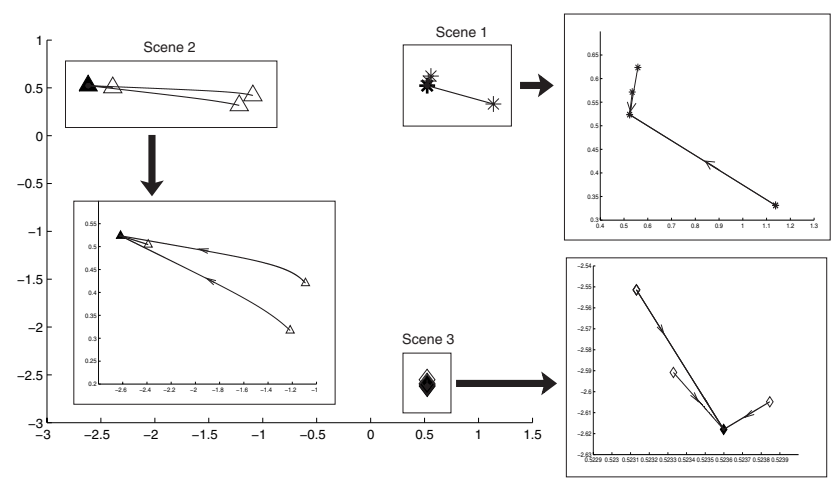

Fig. 5: Tracking moving scenes using third order Kuramoto model. The estimates of $\beta$ cortical inputs at any given time point are mapped to complex pattern in $\mathbb{C}^{3}$ to initialize the phase variables of the Kuramoto model. The phase difference variables converge to one of the three equilibria. For illustration, the points are zoomed.

\section{B. Decoding the location of three stationary targets}

The location of stationary inputs can be identified using statistical hypothesis testing from the associated $\beta$ strands 


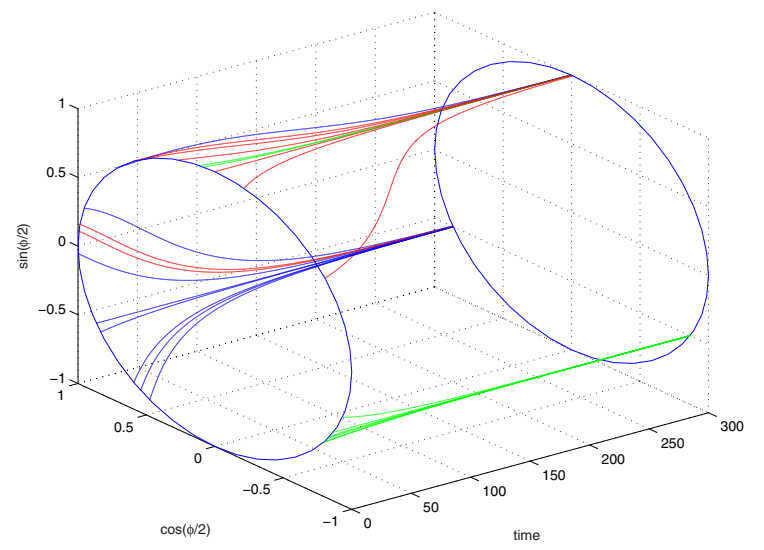

Fig. 6: Detection of input position (left, center and right) with second order Kuramoto model. First 20 principal components of the beta strand at any time point are mapped to complex pattern in $\mathbb{C}^{2}$ to initialize the phase variables of the Kuramoto model. The phase difference variable converge to one of the three equilibria. The figures show the $\cos \left(\frac{\phi}{2}\right)$ and $\sin \left(\frac{\phi}{2}\right)$ over simulation time $s$. Blue, red and green curves are with left, center and right inputs respectively

of the cortical activities by KL-decomposition [4]. In this section we follow this thread and show that the beta strands can also be memorized using the Kuramoto model.

We consider a set of 3 stationary inputs (Left, Center, and Right) and assume that the corresponding $\beta$ strands are already obtained. At any given time, $t$, the strands are given by a vector in $\mathbb{R}^{Q}$ where $Q=20$. Like before, we define a map

$$
T: \mathbb{R}^{Q} \longrightarrow \mathbb{C}^{M}
$$

which maps

$$
v_{i} \longmapsto p_{i}, \quad i=1,2,3
$$

where $M=2$ or 3 , and $v_{i}$ is assumed to be the vector representation of the mean strand and $p_{i}$ are the first three patterns in (17).

For each of the 3 inputs, we obtain multiple simulations of the cortical movie and obtain the associated strands. We initialized Kuramoto model and classify each of the strands and this process has been sketched in Fig. 6 and Fig. 7. The percentage of wrong detection with the third order Kuramoto model has been plotted in Fig. 8, one for each time. It is not surprising to observe that for a longer delay, the probability of error rises.

\section{INITIALIZATION AND RECOGNITION}

Above we illustrated that Kuramoto models can be used to recognize patterns with the proposed mechanism of recognition. The final goal is to implement physically pattern recognition with laser networks. It is easy to set initial values in a computer simulation. But it might be difficult in real laser systems since one does not have direct access to the phase of a laser. One mechanism to set initial values of real laser systems is to entrain the network to a relatively strong external input having appropriate phase relation associated

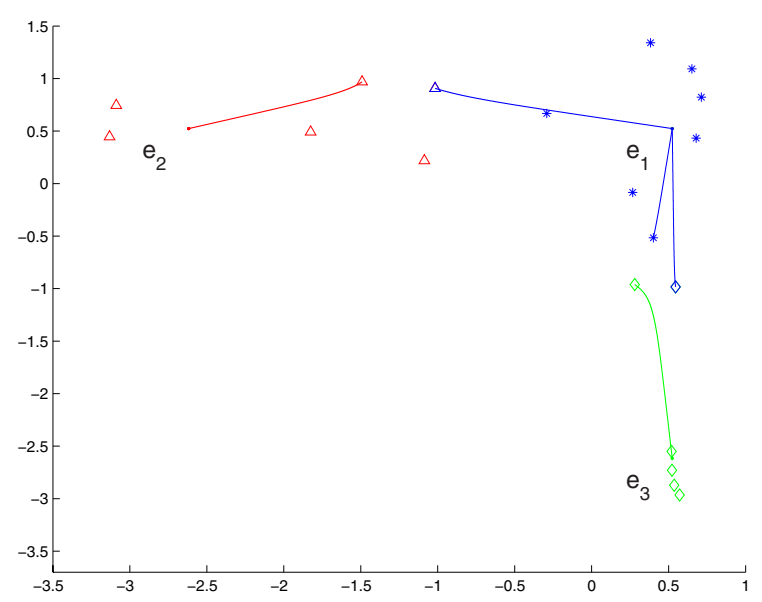

Fig. 7: Detection of input position (left, center and right) with third order Kuramoto model. First 20 principal components of the beta strand at any time point are mapped to complex pattern in $\mathbb{C}^{3}$ to initialize the phase variables of the Kuramoto model. The phase difference variables converge to one of the three equilibria.

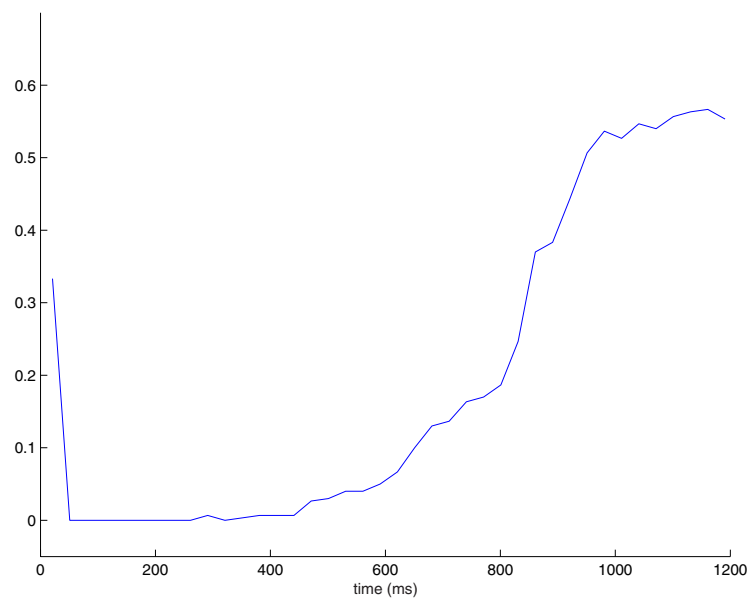

Fig. 8: Detection Error Probability

the recognized patterns [9]. Suppose the vector $v$ to be recognized is mapped into a complex vector $p \in \mathbb{C}^{M}$, we may entrain the network to the associated periodic input $\varepsilon p e^{i \omega t}$, where $\varepsilon$ is much larger than the strength of connections in laser networks, so that the phases phases converge to the desired values. The lasers lock to the signal. After the initialization stage is complete and the lasers have phase relations defined by the vector $v$. We remove the forcing signal. Then the recognition starts from the defined phase relations and the network converges to the appropriate synchronized state. In this paper we only simulated the pattern recognition of three stationary targets with the networks of two lasers in order to show this mechanism to set initial values of lasers. Fig. 9

\section{CONCLUSION}

In conclusion, the weakly connected neural networks where the artificial neurons are lasers provide possible mech- 


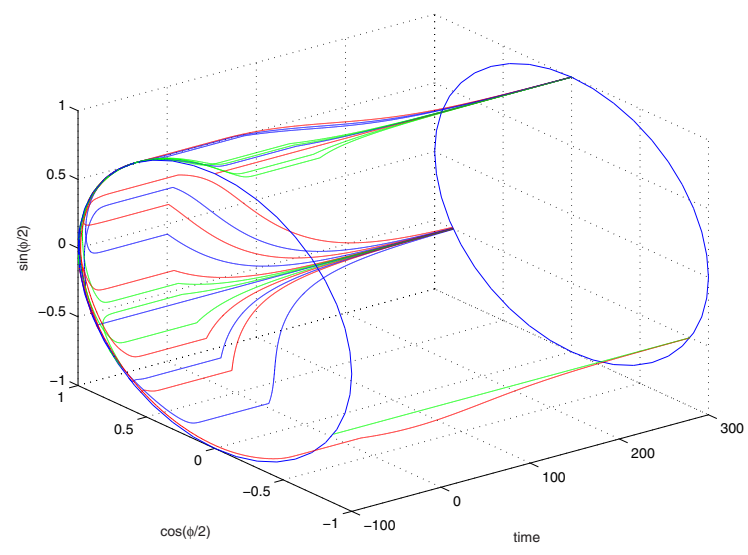

Fig. 9: Detection of input position (left, center and right) with laser network of 2 units initialized by external inputs associated with recognized inputs. The phase difference variable converge to one of the three equilibria. The figures show the $\cos \left(\frac{\phi}{2}\right)$ and $\sin \left(\frac{\phi}{2}\right)$ over simulation time $s$. The time before 0 is the initialization stage and afterwards it is recognition stage. Blue, red and green curves are with left, center and right inputs respectively

anisms of pattern recognition. The mechanism of recognition proposed in this paper can perform pattern recognition with a small number of oscillatory units in neural networks. The prescribed equilibria in phase relations can be easily designed by the connection coefficients.

\section{REFERENCES}

[1] M. A. Arbib, "Brain Theory and Neural Networks", MIT Press, Cambridge, MA, 1995

[2] C. Acker, N. Kopell and J. White, "Synchronization of strongly coupled excitatory neurons: relating network behavior to biophysics," J. Comput. Neurosci., vol. 15, pp. 71-90, 2003

[3] M. Dellnitz, M. Golubitsky and M. Nicol, "Symmetry of attractors and the Karhunen-Loeve Decomposition," in Trends and Perspectives in Applied Mathematics, L. Sirovich, Ed., chapter 4, pp. 73-108, Springer Verlag, New York, 1994.

[4] X. Du, B. K. Ghosh, and P. S. Ulinski, "Encoding and Decoding Target Locations with Waves in the Turtle Visual Cortex", IEEE Transactions on Biomedical Engineering, vol. 52, No. 4, pp. 566-577, 2005.

[5] G. C. Goodwin and K. S. Sin, Adaptive Filtering Prediction and Control, Prentice Hall, 1984.

[6] J. Guckenheimer and P. Holmes, Nonlinear Oscillations, Dynamical Systems, and Bifurcations of Vector Fields, Springer-Verlag, New York, 1997

[7] P. Holmes, J. L. Lumley and G. Berkooz, Turbulence, Coherent Structure, Dynamical Systems and Symmetry, Cambridge University Press, Cambridge, 1996.

[8] F. C. Hoppensteadt and E. M. Izhikevich, Weakly Connected Neural Networks Springer-Verlag, New York, 1997

[9] F. C. Hoppensteadt and E. M. Izhikevich, "Synchronization of Laser Oscillators, Associative Memory, and Optical Neurocomputing", Physical Review E, vol. 62, pp. 4010-4013, 2000

[10] Y. Kuramoto, Chemical Oscillations, Waves, and Turbulence, Springer-Verlag, New York, 1984.

[11] Z. Nenadic, B. K. Ghosh and P. Ulinski, "Modeling and estimation problems in the turtle visual cortex", IEEE Trans. on Biomedical Engineering, vol. 49, no. 8, pp. 753-762, 2002.

[12] Z. Nenadic, B. K. Ghosh and P. Ulinski, "Propagating waves in visual cortex: A large scale model of turtle visual cortex", J. Computational Neuroscience, vol. 14, pp. 161-184, 2003.

[13] H. L. Van Trees, Detection, Estimation and Modulation Theory, John Wiley and Sons, Inc., New York, 1968.
[14] W. Wang, B. K. Ghosh and P. S. Ulinski, "Two Cortical Circuits Control Propagating Waves in Visual Cortex", In press J. Computational Neuroscience, Dec. 2005.

[15] W. Wang, B. K. Ghosh, "Motion Reconstruction in Natural Scenes from Cortical Activity Waves", 16th IFAC World Conference, 2005. 